\title{
Proenkephalin for the Early Detection of Acute Kidney Injury in Hospitalized Patients with Chronic Kidney Disease
}

Tobias Breidthardt,MD ${ }^{1,3}$, Cedric Jaeger,MD²; Andreas Christ,MD; Theresia Klima,MD²; Tamina Mosimann,MD³ Raphael Twerenbold,MD³; Jasper Boeddinghaus, $\mathrm{MD}^{1,3}$; Thomas Nestelberger, $\mathrm{MD}^{3}$; Patrick Badertscher, $\mathrm{MD}^{3}$; Joachim Struck, $\mathrm{PhD}^{4}$; Andreas Bergmann, $\mathrm{PhD}^{4}$; Oliver Hartmann, $\mathrm{PhD}^{4}$; Stefan Kalbermatter,MD5; Giancarlo Marenzi,MD; ${ }^{6}$ Christian Mueller,MD³

\footnotetext{
${ }^{1}$ Division of Internal Medicine, ${ }^{2}$ Department of Nephrology, ${ }^{3}$ Cardiovascular Research Institute Basel (CRIB) and Department of Cardiology, all at the University Hospital Basel, University of Basel, Switzerland; ${ }^{4}$ Sphingotec GmbH Hennigsdorf, Germany; ${ }^{5}$ Department of Nephrology, Kantonsspital Liestal, Liestal, Switzerland; ${ }^{6}$ Centro Cardiologico Monzino, Milan University, Milan, Italy
}

Word count: 2596

Key words: Acute Kidney Injury, Chronic Kidney Disease, Early Detection, Proenkephalin, Sensitivity, Specificity,

Short title: Proenkephalin for the early detection of acute kidney injury

\section{Correspondence to:}

PD Dr. Tobias Breidthardt, Department of Internal Medicine \& CRIB, University Hospital Basel; Petersgraben 4, CH-4031 Basel, Switzerland

Phone: + 416126525 25, Fax; +41 6126553 53, E-mail: $\underline{\text { Tobias.Breidthardt@usb.ch }}$

\section{Funding :}

This study was supported by the University Hospital of Basel, Switzerland and the Swiss National Science Foundation (grant number PP00B-102853). Proenkephalin measurements were performed and provided free of charge by Sphingotec. 


\section{Abstract:}

Background: The early detection of acute kidney injury (AKI) in patients with chronic kidney disease (CKD) is an unmet clinical need. ProEnkephalin (PENK) might improve the early detection of AKI.

Methods: 111 hospitalized CKD patients undergoing radiographic contrast procedures were enrolled. PENK was measured in a blinded fashion at baseline (before contrast media administration) and on day 1 (after contrast media administration). The potential of PENK levels to predict contrast-induced AKI was the primary endpoint.

Results: Baseline creatinine and baseline PENK were similar in AKI and no-AKI patients. In AKI patients day 1 PENK (198pmol/l vs. $121 \mathrm{pmol} / \mathrm{l}, \mathrm{p}<0.01)$ was significantly higher compared to no-AKI patients. The area under the curve (AUC) for the prediction of AKI by day 1 PENK was $0.79,95 \% \mathrm{Cl} 0.70-0.87$, similar to serum creatinine: $0.78,95 \% \mathrm{Cl} 0.61-0.95$. Delta PENK was significantly higher in AKI compared to no-AKI patients (53pmol// vs. $1 \mathrm{pmol} / \mathrm{l}, \mathrm{p}<0.01)$. The $A U C$ for the prediction of AKI by delta PENK was high $(0.92,95 \% \mathrm{Cl} 0.82-1.00)$ and remained high for creatinine-blind AKI $(0.94,95 \% \mathrm{Cl} 0.87-0.97)$.

Conclusion: Delta PENK levels improve the early detection of contrast-induced AKI in CKD patients over serial creatinine sampling. Delta PENK accelerates the detection of creatinine-blind AKI by 24 hours. 


\section{Introduction:}

Acute kidney injury (AKI) is a clinical syndrome defined by a sudden decline in glomerular filtration rate affecting about $5 \%$ of all hospitalized patients ${ }^{1}$. Its incidence has steadily increased over the last decade ${ }^{2}$ and estimates assume that annually about 17 million hospital admissions in the United States are complicated by AKI ${ }^{3}$. lodinated contrast media $(\mathrm{CM})$ administration is one of the most important triggers of AKI ${ }^{4}$ and contrast-induced kidney injury has repeatedly been linked to increased morbidity, mortality and treatment costs ${ }^{5-7}$. Hence, the accurate early detection of AKI is an unmet clinical need.

The novel biomarker ProEnkephalin (PENK) is a stable fragment derived from the precursor Enkephalins, which are known as small endogenous opioid peptides and are produced throughout the human body, including the kidneys ${ }^{8}$. Spot measurements of PENK were recently shown to reflect renal function in healthy subjects and elevated levels are independently associated with an accelerated decline in renal function and a progression to chronic kidney disease ${ }^{9}$. However, PENK is a dynamic parameter and its levels can significantly change within hours. For example, a small pilot study recently showed PENK levels to increase within 6 hours after cardiac surgery in patients experiencing post-surgical AKI, while simultaneously measured serum creatinine levels showed a delayed increase ${ }^{10}$. Hence, these data suggest that PENK levels might improve the early detection of AKI ${ }^{10,11}$.

These previous studies predominantly included patients with preserved renal function, despite chronic kidney disease (CKD) being a powerful risk factor for the development of $\mathrm{AKI}{ }^{12,13}$. Hence, any biomarker aiming to improve the early detection of $\mathrm{AKI}$ in clinical practice needs to retain its diagnostic potential in the vulnerable CKD patient population. We therefore aimed to examine the potential of PENK levels to improve 
the early detection of $\mathrm{AKI}$ over the current clinical gold-standard of serial serum creatinine sampling in hospitalized CKD patients. 


\section{Methods:}

This study uses prospectively collected plasma obtained in a multicenter, randomized, open-label, controlled trial (Clinicaltrials.gov Identifier: NCT00130598) that compared two regimens of sodium bicarbonate vs. standard volume supplementation with isotonic sodium chloride in the prevention of contrast-induced AKI in hospitalized patients with chronic renal dysfunction. The study was conducted between March 2005 and December 2009 and its details have been described before ${ }^{14}$. In short, renal dysfunction was defined as serum creatinine level $>93 \mu \mathrm{mol} / L$ for women and $>117$ $\mu \mathrm{mol} / \mathrm{L}$ for men or an estimated glomerular filtration rate (eGFR) $<60 \mathrm{~mL} / \mathrm{min} / 1.73 \mathrm{~m}^{2}$ (assessed 24 hours before the radiographic procedure). Patients were excluded if they were $<18$ years old, pregnant, or allergic to radiographic contrast, were undergoing dialysis, had severe heart failure (NYHA III-IV), had taken N-acetylcysteine $\leq 24$ hours before $\mathrm{CM}$ administration, or suffered from a vulnerable condition requiring continuous fluid therapy (e.g. severe sepsis). For this study only patients with complete baselineday 1 plasma sample pairs were included. The study was conducted according to the principles of the revised Declaration of Helsinki, had been approved by the local ethical committees and all participants gave written informed consent for the study. The authors designed the study, gathered, analyzed and report the data according to the STARD guidelines for studies of diagnostic accuracy (Table S1 Supplementary Appendix) and made the decision to submit it for publication. TB, CJ and CM had full access to all of the data in the study and vouch for the accuracy and completeness of the data, the analysis, and the presentation, take responsibility for the integrity of the data and the accuracy of the data analysis. TB and CM wrote the first draft of the manuscript. 


\section{Biomarker Measurements}

Plasma samples were prospectively collected at baseline and Day 1. After centrifugation, samples were frozen at $-80^{\circ} \mathrm{C}$ until assayed in a blinded fashion in a single batch. The assay for stable PENK (molecular weight 4,586 Da) was previously described ${ }^{15}$, and has since been modified ${ }^{16}$ (sphingotest ${ }^{\boxplus}$ penKid). In brief, 2 mouse monoclonal anti-PENK antibodies were developed by immunization with PENK peptide. Standards or samples $(50 \mu$ l plasma) were immobilized by the capture antibody ( $2 \mu \mathrm{g}$ coated on polystyrene tubes). The detector antibody was labeled with methylacridinium ester, and bound chemiluminescence was measured. The normal range was mean \pm SEM of $46.6 \pm 14.1 \mathrm{pmol} / \mathrm{l}$, with a median of $45 \mathrm{pmol} / /$ (range 9 to $518 \mathrm{pmol} / \mathrm{l}){ }^{15}$. Creatinine measurements were performed on a Roche Modular P1 analyzer with the enzymatic Creatinine-PAP method for quantification (Roche Diagnostics, Rotkreuz, Switzerland). The estimated glomerular filtration rate (eGFR) was calculated using the abbreviated Modification of Diet in Renal Disease Study (MDRD) equation ${ }^{17}$.

\section{Endpoint}

The ability of PENK levels to detect the occurrence of contrast-induced AKI was assessed as the primary endpoint. Development of contrast-induced AKI was defined as a serum creatinine increase of $\geq 25 \%$ or an increase of $\geq 44 \mu \mathrm{mol} / \mathrm{L}$ over the baseline serum creatinine concentration within the first $48 \mathrm{~h}$ after $\mathrm{CM}$ administration ${ }^{14,18}$. Creatinine-blind AKI was defined as AKI occurring between day 1 and day 2, i.e. not detectable by creatinine at day 1 . Creatinine-blind AKI was considered as a secondary endpoint. 


\section{Statistical analysis}

The statistical analyses were performed using the SPSS/PC (version 22.0, IBM SPSS, IL, USA) and MedCalc (version 9.2., MedCalc, Ostend, Belgium) software packages. A statistical significance level of 0.05 was used. All hypothesis testing was two-tailed. Discrete variables are expressed as counts (percentage) and continuous variables as means \pm standard deviation (SD) or median and interquartile range [IQR], unless stated otherwise. The comparison between the two groups was done using chisquare test, Fishers' exact test, t-test, Mann-Whitney $U$ or Wilcoxon test as appropriate. Spearman rank correlation was used to perform correlation analyses. The prognostic accuracy of the different models was evaluated using receiver operating characteristic $(\mathrm{ROC})$ curve analysis. Univariate binary regression analysis was applied to identify predictors of contrast-induced AKI. 


\section{Results}

\section{Study Population}

A patient flow chart for the current study is displayed in Figure 1. Detailed characteristics of study population are summarized in Table 1 . The median age of the 111 patients was 77 years (IQR 72-80). Most patients had stage 3 CKD with a median eGFR at baseline of $45 \mathrm{~mL} / \mathrm{min} / 1.73 \mathrm{~m}^{2}$ (IQR 38-50). Hypertensive nephropathy was the main aetiology of CKD (41\%), followed by combined hypertensive and diabetic nephropathy (26\%) and other not further specified nephropathies (18\%). The majority of patients underwent either computer tomography scans $(50 \%)$ or cardiac catheterization with (17\%) or without PCl (21\%). A median of $100 \mathrm{ml}$ of CM (IQR 70140) was administered during contrast procedures. Overall, 7 of 111 patients (6\%) developed contrast-induced AKI.

\section{Baseline ProEnkephalin levels}

Baseline PENK levels were moderately correlated to baseline creatinine $(r=0.27$; $p<0.01)$, heart rate $(r=0.22 ; p=0.02)$ and body mass index $(r=-0.23 ; p=0.02)$, but not to age $(p=0.18)$ and systolic blood pressure $(p=0.31)$. The median PENK level in the overall patient population was 126pmol/I [IQR 100-166] and 97 patients (87\%) displayed PENK levels above the upper limit of normal (80pmol/L); 80 patients $(75 \%)$ displayed PENK levels above 100pmol/L. As shown in table 1, baseline characteristics were well balanced between patients with or without subsequent AKI. Baseline creatinine (141umol// [IQR: 137-164] vs. 133umol [IQR: 109-166], $\mathrm{p}=0.65$ ), and baseline PENK (166pmol// [IQR: 87-237] vs. 124pmol/I [IQR: 100-162], p=0.36) were similar in AKI and no-AKI patients (Figure 2a). Consequently, the AUC for the prediction of contrast-induced AKI was poor for baseline PENK $(0.60,95 \% \mathrm{Cl} 0.34$ - 
$0.86)$ and baseline creatinine $(0.55,95 \% \mathrm{Cl} 0.37-0.74)$ (Figure 3$)$. In binary regression analyses both biomarkers baseline values failed to predict the subsequent occurrence of $\mathrm{AKI}$ ( $\mathrm{p}=0.69$ and 0.72 , respectively). A secondary analysis applied the serum criteria of the KDIGO AKI classification; 8 out of 111 patients developed contrast-induced KDIGO AKI. In this secondary analysis baseline PENK achieved a similar AUC (0.63, $95 \% \mathrm{Cl} 0.40-0.87)$ for the prediction of contrast-induced KDIGO AKI.

\section{Serial ProEnkephalin levels for the prediction of contrast-induced AKI}

In patients experiencing contrast-induced AKI, PENK levels (198pmol/I [143-293] vs. $121 \mathrm{pmol} / \mathrm{l}$ [92-169], $\mathrm{p}<0.01)$ at day 1 were significantly higher compared to no-AKI patients (Figure 2b). To evaluate the potential of day 1 PENK levels to detect the occurrence of AKI, ROC analyses were performed. The AUC for the prediction of AKI was: $0.79,95 \% \mathrm{Cl} 0.70-0.87$, similar to the AUC obtained by serum creatinine: 0.78 , 95\% $\mathrm{Cl}$ 0.61-0.95 (Figure 3). In a secondary analysis, which applied the serum criteria of the KDIGO AKI classification day 1 PENK achieved a similar AUC $(0.81,95 \% \mathrm{Cl}$ 0.69-0.94) for the prediction of contrast-induced KDIGO AKI.

\section{ProEnkephalin levels for the prediction of creatinine-blind AKI}

Overall, 5 of the $7 \mathrm{AKI}$ cases were already detectable by day 1 serum creatinine. When restricting the analysis to creatinine-blind AKI cases, occurring between day 1 and day 2, day 1 PENK achieved a AUC of $0.94(95 \% \mathrm{Cl} 0.88-0.98)$ for the prediction of late AKI, while the AUC for day 1 serum creatinine was only moderate $(0.72,95 \% \mathrm{Cl} 0.54$ 0.91). When entering day 1 biomarker levels into a binary regression analysis, day 1 PENK levels detected overall AKI (OR per standard deviation increase: $1.71,95 \% \mathrm{Cl}$ 1.02-2.87, $\mathrm{p}=0.04$ ) but did not significantly predict creatinine-blind AKI (OR per standard deviation increase: $1.89,95 \% \mathrm{Cl} 0.91-3.87, \mathrm{p}=0.08)$. Similarly, day 1 serum 
creatinine levels were associated with overall AKI (OR per standard deviation increase: $1.75,95 \% \mathrm{Cl} 1.02-3.01, \mathrm{p}=0.04)$ but not with creatinine-blind AKI (OR per standard deviation increase: $1.19,95 \% \mathrm{Cl} 0.36-3.88, \mathrm{p}=0.78)$.

\section{Delta ProEnkephalin for the prediction of contrast-induced AKI}

In the overall patient population, there was no change between baseline and day 1 PENK levels (median change: $2.23 \mathrm{pmol} / \mathrm{l}, \mathrm{p}=0.47$ ). However, delta PENK was significantly higher in AKI compared to no-AKI patients (53pmol// [36-83] vs. 1pmol// [14 - 17], $\mathrm{p}<0.01$ ) (Figure 2c). Delta PENK was moderately correlated to the maximal serum 48 h creatinine increase $(r=0.24, p=0.01)$ but not to baseline PENK $(p=0.09)$, or baseline serum creatinine $(p=0.61)$. To evaluate the potential of delta PENK to predict the occurrence of AKI, ROC analyses were performed. The AUC for the prediction of AKI by delta PENK was high: $0.92,95 \% \mathrm{Cl}$ 0.82-1.00 (Figure 3). In a secondary analysis, which applied the serum criteria of the KDIGO AKI classification delta PENK achieved a similar AUC $(0.93,95 \% \mathrm{Cl} 0.84-1.00)$ for the prediction of contrast-induced KDIGO AKI. When restricting the analysis to creatinine-blind AKI cases, occurring between day 1 and day 2, delta serum creatinine only achieved a moderate AUC (AUC $0.65,95 \% \mathrm{Cl} 0.23-1.00)$. In contrast the predictive potential of delta PENK for creatinine-blind AKI (AUC 0.94, 95\% $\mathrm{Cl} 0.87-0.97$ ) remained high. When entering the biomarkers into a univariate binary regression analysis delta creatinine (OR per standard deviation increase: $15.78,95 \% \mathrm{Cl} 2.88-86.58 ; \mathrm{p}<0.01$ ) and delta PENK (OR per standard deviation increase: $6.60,95 \% \mathrm{Cl} 2.25-19.35 ; \mathrm{p}=0.01)$ predicted the occurrence of overall AKI. In contrast, age $(p=0.85)$, gender $(p=0.25)$, BMI $(p=0.25)$, systolic blood pressure (0.28), steady state renal function (eGFR $p=0.98$ ), medical therapy (ACE-I $p=0.10 ; A R B p=0.11$; diuretics $p=0.49)$ and the volume of $C M(p=0.96)$ all were not suitable to predict the occurrence of AKI. When restricting the regression 
analysis to creatinine-blind AKI cases, occurring between day 1 and day 2, only delta PENK remained significantly associated with the occurrence of late AKI (OR per standard deviation increase: $3.13,95 \% \mathrm{Cl} 1.02-9.64, \mathrm{p}=0.04$ ). 


\section{Discussion}

In this investigation we examined the potential of PENK levels at baseline and serially thereafter to predict the occurrence of contrast-induced AKI in 111 hospitalized CKD patients. We report five major findings. First, baseline PENK levels drawn before the administration of $\mathrm{CM}$ were significantly correlated to steady state renal function. Second, baseline PENK and baseline creatinine levels were similar in AKI and no-AKI patients and failed to predict the subsequent occurrence of contrast-induced AKI. The similarity of baseline serum creatinine in AKI and no-AKI patients is probably due to the selective inclusion of CKD patients into our study. Since CKD is the most common risk factor for $\mathrm{CIN}{ }^{12,13}$, studies enrolling patients irrespective of prior renal function frequently describe higher baseline creatinine levels in patients subsequently developing CIN 10,11. Third, PENK levels increased significantly after the administration of $\mathrm{CM}$ in patients developing contrast-induced $\mathrm{AKI}$, while remaining stable in no-AKI patients. Consequently, day 1 PENK levels after CM administration were significantly higher in AKI compared to no-AKI patients. Forth, Day 1 PENK and serum creatinine levels detected overall AKI with similar accuracy. Fifth, delta PENK achieved the best diagnostic accuracy for the detection of overall AKI and creatinineblind AKI, providing AUCs of 0.92 and 0.94, respectively.

Our results extend and corroborate previous studies investigating the potential clinical applicability of PENK. The correlation of baseline PENK with concurrently drawn serum creatinine values has been described before ${ }^{10,15,19}$. This link between baseline PENK levels and steady state renal function is further highlighted by the strong association of published PENK levels with steady state renal function described across a wide range of populations, which appear to show a near linear increase of 
PENK with decreasing renal function (i.e. healthy participants: eGFR $91 \mathrm{ml} / \mathrm{min}$, PENK 46pmol/L" cardiac surgery: eGFR 75ml/min, PENK 85pmol/L ${ }^{11}$; acute heart failure: eGFR 56ml/min, PENK 97pmol//19; CKD current study: eGFR 45ml/min, PENK 126pmol/l).

This link between chronic renal function and baseline PENK levels might also explain the apparent disagreement between our results and two recent studies investigating patients undergoing cardiac surgery ${ }^{10,11}$. These studies found baseline PENK levels drawn before surgery to be increased in patients suffering from postsurgical AKI 10,11. However, both studies showed pronounced differences in the prevalence of CKD between AKI and no-AKI patients, with CKD being four times more common in $\mathrm{AKI}$ compared to the no-AKI patients. We therefore believe that the increased baseline PENK levels observed for AKI patients mainly represent impaired chronic renal function, rather than an early $\mathrm{AKI}$ signal. This interpretation is backed by Mossanen and co-workers, who found the diagnostic accuracy of baseline PENK levels to decrease after the exclusion of patients with known CKD and subsequently elevated baseline PENK levels ${ }^{11}$. Importantly, both studies found preoperative PENK levels to not improve the prediction of AKI over baseline serum creatinine ${ }^{10,11}$.

In this CKD cohort, the vast majority of patients displayed PENK levels above the previously suggested universal cut-off value of 100pmol/L but did not develop subsequent $\mathrm{AKI}$. This observation might at least partially be caused by PENK accumulation in CKD patients and the strong association of PENK with chronic renal dysfunction. It highlights the need for further studies to establish clinically applicable cut-off values in CKD patients.

The early PENK increase in patients experiencing AKI compared to the stable values in no-AKI patients observed in the current study suggests delta PENK as 
powerful marker for the early detection of AKI. Importantly, delta PENK was strongly associated with overall $\mathrm{AKI}$ and retained its diagnostic accuracy for the detection of creatinine-blind AKI, thereby accelerating the clinical diagnosis of creatinine-blind AKI by 24 hours. The diagnostic potential of early PENK changes was also observed by Shah and co-workers, who assessed 92 patients undergoing cardiac surgery ${ }^{10}$ and also found delta PENK to best predict late AKI.

Despite the lack of causative therapeutic interventions currently available for the treatment of $\mathrm{AKI}$ the early detection of $\mathrm{AKI}$ is of vital importance and offers a window of opportunity for the initiation of supportive measures (i.e. volume management, maintenance of adequate blood pressure, avoidance of nephrotoxins) to reduce the duration of the AKI episode ${ }^{20,21}$. The enormous potential of early supportive therapy was recently highlighted in a study assessing the impact of electronic automated AKI alerts. This study showed a convincing 7 -fold decrease in the requirement of renal replacement therapy and a 2.4 -fold decrease in mortality in patients receiving supportive therapy on the day of the alert compared to patients receiving delayed supportive therapy (day 1 or later) ${ }^{22}$. Of note, this intervention was based on the review of serial creatinine measurements and the additional 24 hour window of opportunity provided delta PENK could potentially provide even bigger benefits ${ }^{23}$.

Potential limitations of the current study merit consideration. First, our study has a moderate number of patients and only few developed AKI. However, the strong correlation of baseline PENK with stable serum creatinine values observed in our study is in line with various other publications ${ }^{10,15,19}$. We are therefore confident, that our baseline PENK results will apply to other populations. Nevertheless, due to the low number of AKI cases observed in our cohort, we cannot exclude the possibility of a Type II error for the serial sampling part of our study. Second, we did not include the 
any urine output criteria into the definition of contrast-induced AKI. However, while recent data suggest, that including urine output criteria into the AKI definition might increase the AKI incidence ${ }^{24}$, most previously published studies assessing the predictive potential of AKI biomarkers in the non-ICU setting, also opted to exclude the urine output criteria for AKI diagnosis ${ }^{10,15,25}$ since measuring urine output in a non-ICU setting is tedious and error-prone. When routinely measuring urine output via a urinary catheter any additional information obtained by measuring urine output needs to be carefully weighed against the potential morbidity, mortality, and healthcare expenditure associated with catheter-associated urinary tract infections ${ }^{26}$. Third, a general limitation for the comparison of a novel marker of interest for the prediction of AKI with creatinine is that this is principally biased by the fact that creatinine is a constituent of the AKI definition.

\section{Conclusion:}

Delta PENK levels improve may the early detection of contrast-induced AKI over serial creatinine sampling. Delta PENK may accelerate the detection of creatinine-blind AKI by 24 hours. 


\section{Conflict of Interest Statement}

Dr. Breidthardt has received research grants from the Swiss National Science Foundation (PASMP3-134362), the University Hospital Basel, the Department of Internal Medicine, University Hospital Basel, Abbott and Roche as well as speakers honoraria from Roche and Novartis. Dr. Twerenbold has received speaker honoraria from Roche, as well as a research grant from the Swiss National Science Foundation. Dr. Boeddinghaus received research grants from the University of Basel and the Division of Internal Medicine and speaker honoraria from Siemens. Professor Mueller has received research grants from the Swiss National Science Foundation and the Swiss Heart Foundation, the European Union, the Cardiovascular Research Foundation Basel, the University of Basel, 8sense, Abbott, ALERE, Astra Zeneca, Beckman Coulter, Biomerieux, BRAHMS, Critical Diagnostics, Nanosphere, Roche, Siemens, Singulex, and the University Hospital Basel, as well as speaker or consulting honoraria from Abbott, ALERE, Astra Zeneca, BG Medicine, Biomerieux, BMS, Boehringer Ingelheim, BRAHMS, Cardiorentis, Daiichi Sankyo, Novartis, Roche, Sanofi, Singulex, and Siemens. Dr. Bergmann is CEO and shareholder of sphingotec GmbH; Drs. Hartmann and Struck are employed by sphingotec $\mathrm{GmbH}$. The other authors report no conflicts. 


\section{References:}

1. Selby NM, Crowley L, Fluck RJ, et al. Use of electronic results reporting to diagnose and monitor AKI in hospitalized patients. Clinical journal of the American Society of Nephrology : CJASN. 2012;7(4):533-540.

2. Hsu CY, McCulloch CE, Fan D, Ordonez JD, Chertow GM, Go AS. Community-based incidence of acute renal failure. Kidney Int. 2007;72(2):208212.

3. Chertow GM, Burdick E, Honour M, Bonventre JV, Bates DW. Acute kidney injury, mortality, length of stay, and costs in hospitalized patients. J Am Soc Nephrol. 2005;16(11):3365-3370.

4. Tublin ME, Murphy ME, Tessler FN. Current concepts in contrast mediainduced nephropathy. AJR Am J Roentgenol. 1998;171(4):933-939.

5. Finn WF. The clinical and renal consequences of contrast-induced nephropathy. Nephrol Dial Transplant. 2006;21(6):i2-10.

6. McCullough PA. Contrast-induced acute kidney injury. J Am Coll Cardiol. 2008;51(15):1419-1428.

7. Rihal CS, Textor SC, Grill DE, et al. Incidence and prognostic importance of acute renal failure after percutaneous coronary intervention. Circulation. 2002;105(19):2259-2264.

8. Denning GM, Ackermann LW, Barna TJ, et al. Proenkephalin expression and enkephalin release are widely observed in non-neuronal tissues. Peptides. 2008;29(1):83-92.

9. Schulz CA, Christensson A, Ericson U, et al. High Level of Fasting Plasma Proenkephalin-A Predicts Deterioration of Kidney Function and Incidence of CKD. J Am Soc Nephrol. 2017;28(1):291-303.

10. Shah KS, Taub $P$, Patel $M$, et al. Proenkephalin predicts acute kidney injury in cardiac surgery patients. Clin Nephrol. 2015;83(1):29-35.

11. Mossanen JC, Pracht J, Jansen TU, et al. Elevated Soluble Urokinase Plasminogen Activator Receptor and Proenkephalin Serum Levels Predict the Development of Acute Kidney Injury after Cardiac Surgery. Int J Mol Sci. 2017;18(8).

12. Hsu RK, Hsu CY. The Role of Acute Kidney Injury in Chronic Kidney Disease. Semin Nephrol. 2016;36(4):283-292.

13. Rear R, Bell RM, Hausenloy DJ. Contrast-induced nephropathy following angiography and cardiac interventions. Heart. 2016;102(8):638-648.

14. Klima T, Christ A, Marana I, et al. Sodium chloride vs. sodium bicarbonate for the prevention of contrast medium-induced nephropathy: a randomized controlled trial. Eur Heart J. 2012;33(16):2071-2079.

15. Marino R, Struck J, Hartmann O, et al. Diagnostic and short-term prognostic utility of plasma pro-enkephalin (pro-ENK) for acute kidney injury in patients admitted with sepsis in the emergency department. $J$ Nephrol. 2015;28(6):717-724.

16. $\mathrm{Ng} \mathrm{LL}$, Sandhu JK, Narayan $\mathrm{H}$, et al. Proenkephalin and prognosis after acute myocardial infarction. J Am Coll Cardiol. 2014;63(3):280-289.

17. Levey AS, Bosch JP, Lewis JB, Greene T, Rogers N, Roth D. A more accurate method to estimate glomerular filtration rate from serum creatinine: a new prediction equation. Modification of Diet in Renal Disease Study Group. Ann Intern Med. 1999;130(6):461-470. 
18. Ozkok S, Ozkok A. Contrast-induced acute kidney injury: A review of practical points. World J Nephrol. 2017;6(3):86-99.

19. $\mathrm{Ng}$ LL, Squire IB, Jones DJ, et al. Proenkephalin, Renal Dysfunction, and Prognosis in Patients With Acute Heart Failure: A GREAT Network Study. $J$ Am Coll Cardiol. 2017;69(1):56-69.

20. Brown JR, Kramer RS, Coca SG, Parikh CR. The prognostic value of using the duration of acute kidney injury in cardiac surgery: an example using two antifibrinolytics. J Extra Corpor Technol. 2011;43(4):227-231.

21. Coca SG, King JT, Jr., Rosenthal RA, Perkal MF, Parikh CR. The duration of postoperative acute kidney injury is an additional parameter predicting longterm survival in diabetic veterans. Kidney Int. 2010;78(9):926-933.

22. Prendecki M, Blacker E, Sadeghi-Alavijeh O, et al. Improving outcomes in patients with Acute Kidney Injury: the impact of hospital based automated AKI alerts. Postgrad Med J. 2016;92(1083):9-13.

23. Meersch M, Schmidt C, Hoffmeier A, et al. Prevention of cardiac surgeryassociated AKI by implementing the KDIGO guidelines in high risk patients identified by biomarkers: the PrevAKI randomized controlled trial. Intensive Care Med. 2017;43(11):1551-1561.

24. Shacham $Y$, Rofe M, Leshem-Rubinow E, et al. Usefulness of urine output criteria for early detection of acute kidney injury after transcatheter aortic valve implantation. Cardiorenal Med. 2014;4(3-4):155-160.

25. Nickolas TL, O'Rourke MJ, Yang J, et al. Sensitivity and specificity of a single emergency department measurement of urinary neutrophil gelatinaseassociated lipocalin for diagnosing acute kidney injury. Ann Intern Med. 2008;148(11):810-819.

26. Saint S, Meddings JA, Calfee D, Kowalski CP, Krein SL. Catheter-associated urinary tract infection and the Medicare rule changes. Ann Intern Med. 2009;150(12):877-884. 


\section{Table 1: Baseline Characteristics}

\begin{tabular}{|c|c|c|c|c|}
\hline Total & $\begin{array}{l}\text { Overall } \\
(n=111)\end{array}$ & $\begin{array}{c}\text { AKI } \\
(n=7)\end{array}$ & $\begin{array}{l}\text { No AKI } \\
(n=104)\end{array}$ & $p$ value \\
\hline Sex (male) & $69(62)$ & $3(42)$ & $66(64)$ & 0.53 \\
\hline Age (years) & 77 [72-80] & 78 [68-85] & 76 [67-81] & 0.82 \\
\hline \multicolumn{5}{|l|}{ Comorbidities: } \\
\hline Diabetes mellitus - no. (\%) & $48(43 \%)$ & $4(57 \%)$ & $44(42 \%)$ & 0.23 \\
\hline Hypertension - no. (\%) & $94(85)$ & $6(100)$ & $88(85)$ & 0.30 \\
\hline Coronary Heart Disease - no. (\%) & $65(59)$ & $4(57)$ & $61(59)$ & 0.68 \\
\hline Congestive Heart Failure - no. (\%) & $37(33)$ & $2(29)$ & $45(43)$ & 0.60 \\
\hline Peripheral Artery Disease - no. (\%) & $30(27)$ & $0(0)$ & $30(29)$ & 0.13 \\
\hline COPD - no. (\%) & $29(26)$ & $2(29)$ & $27(26)$ & 0.68 \\
\hline Hypercholesterinemia - no. (\%) & $66(60)$ & $2(29)$ & $64(62)$ & 017 \\
\hline \multicolumn{5}{|l|}{ Nephropathy: } \\
\hline $\begin{array}{l}\text { Hypertensive Nephropathy- no. } \\
(\%)\end{array}$ & $45(41))$ & $2(33)$ & $43(41)$ & 0.71 \\
\hline Diabetic Nephropathy - no. (\%) & $9(8)$ & $1(17)$ & $8(8)$ & 0.43 \\
\hline $\begin{array}{l}\text { Hypertensive and Diabetic } \\
\text { Nephropathy - no. (\%) }\end{array}$ & $29(26)$ & $2(33)$ & $27(25)$ & 0.68 \\
\hline Other Nephropathy- no. (\%) & $20(18)$ & $1(17)$ & $19(18)$ & 0.93 \\
\hline eGFR (MDRD) baseline (ml/min) & $45[38-50]$ & $41[32-46]$ & 45 [34-53] & 0.64 \\
\hline \multicolumn{5}{|l|}{ Medication: } \\
\hline ACEI/ARB - no. (\%) & $86(77)$ & $5(71)$ & $51(78)$ & 0.64 \\
\hline Diuretics - no. (\%) & $81(73)$ & $6(86)$ & $75(72)$ & 0.58 \\
\hline Statins - no. (\%) & $52(47)$ & $2(29)$ & $50(48)$ & 0.48 \\
\hline Oral Antidiabetics - no. (\%) & $23(21)$ & $2(29)$ & $21(20)$ & 0.44 \\
\hline Aspirin - no. (\%) & $71(64)$ & $5(71)$ & $66(63)$ & 0.32 \\
\hline \multicolumn{5}{|l|}{ Intervention: } \\
\hline amount of contrast (ml) & $100[70-140]$ & $100[70-100]$ & 100 [70-140] & 0.99 \\
\hline
\end{tabular}




\begin{tabular}{|c|c|c|c|}
\hline \multicolumn{4}{|c|}{ Table S1: STARD checklist for reporting of studies of diagnostic accuracy } \\
\hline Section and Topic & $\begin{array}{c}\text { Item } \\
\#\end{array}$ & & On page \# \\
\hline $\begin{array}{l}\text { TITLE/ABSTRACT/ } \\
\text { KEYWORDS }\end{array}$ & 1 & $\begin{array}{l}\text { Identify the article as a study of diagnostic accuracy (recommend MeSH } \\
\text { heading 'sensitivity and specificity'). }\end{array}$ & 1 \\
\hline INTRODUCTION & 2 & $\begin{array}{l}\text { State the research questions or study aims, such as estimating diagnostic } \\
\text { accuracy or comparing accuracy between tests or across participant } \\
\text { groups. }\end{array}$ & 3,4 \\
\hline \multicolumn{4}{|c|}{ ( } \\
\hline \multirow[t]{4}{*}{ Participants } & 3 & $\begin{array}{l}\text { The study population: The inclusion and exclusion criteria, setting and } \\
\text { locations where data were collected. }\end{array}$ & 5 \\
\hline & 4 & $\begin{array}{l}\text { Participant recruitment: Was recruitment based on presenting symptoms, } \\
\text { results from previous tests, or the fact that the participants had received } \\
\text { the index tests or the reference standard? }\end{array}$ & 5 \\
\hline & 5 & $\begin{array}{l}\text { Participant sampling: Was the study population a consecutive series of } \\
\text { participants defined by the selection criteria in item } 3 \text { and } 4 \text { ? If not, } \\
\text { specify how participants were further selected. }\end{array}$ & 5 \\
\hline & 6 & $\begin{array}{l}\text { Data collection: Was data collection planned before the index test and } \\
\text { reference standard were performed (prospective study) or after } \\
\text { (retrospective study)? }\end{array}$ & 5 \\
\hline \multirow[t]{5}{*}{ Test methods } & 7 & The reference standard and its rationale. & 6 \\
\hline & 8 & $\begin{array}{l}\text { Technical specifications of material and methods involved including how } \\
\text { and when measurements were taken, and/or cite references for index } \\
\text { tests and reference standard. }\end{array}$ & 6 \\
\hline & 9 & $\begin{array}{l}\text { Definition of and rationale for the units, cut-offs and/or categories of the } \\
\text { results of the index tests and the reference standard. }\end{array}$ & 6 \\
\hline & 10 & $\begin{array}{l}\text { The number, training and expertise of the persons executing and reading } \\
\text { the index tests and the reference standard. }\end{array}$ & 6 \\
\hline & 11 & $\begin{array}{l}\text { Whether or not the readers of the index tests and reference standard } \\
\text { were blind (masked) to the results of the other test and describe any } \\
\text { other clinical information available to the readers. }\end{array}$ & 6 \\
\hline \multirow[t]{2}{*}{ Statistical methods } & 12 & $\begin{array}{l}\text { Methods for calculating or comparing measures of diagnostic accuracy, } \\
\text { and the statistical methods used to quantify uncertainty (e.g. } 95 \% \\
\text { confidence intervals). }\end{array}$ & 7 \\
\hline & 13 & Methods for calculating test reproducibility, if done. & na \\
\hline \multicolumn{4}{|l|}{ RESULTS } \\
\hline \multirow[t]{3}{*}{ Participants } & 14 & $\begin{array}{l}\text { When study was performed, including beginning and end dates of } \\
\text { recruitment. }\end{array}$ & 5 \\
\hline & 15 & $\begin{array}{l}\text { Clinical and demographic characteristics of the study population (at least } \\
\text { information on age, gender, spectrum of presenting symptoms). }\end{array}$ & $\begin{array}{l}8, \\
\text { Table } 1\end{array}$ \\
\hline & 16 & $\begin{array}{l}\text { The number of participants satisfying the criteria for inclusion who did or } \\
\text { did not undergo the index tests and/or the reference standard; describe } \\
\text { why participants failed to undergo either test (a flow diagram is strongly } \\
\text { recommended). }\end{array}$ & Figure 1 \\
\hline \multirow[t]{4}{*}{ Test results } & 17 & $\begin{array}{l}\text { Time-interval between the index tests and the reference standard, and } \\
\text { any treatment administered in between. }\end{array}$ & n.a. \\
\hline & 18 & $\begin{array}{l}\text { Distribution of severity of disease (define criteria) in those with the target } \\
\text { condition; other diagnoses in participants without the target condition. }\end{array}$ & n.a. \\
\hline & 19 & $\begin{array}{l}\text { A cross tabulation of the results of the index tests (including } \\
\text { indeterminate and missing results) by the results of the reference } \\
\text { standard; for continuous results, the distribution of the test results by the } \\
\text { results of the reference standard. }\end{array}$ & Figure 3 \\
\hline & 20 & $\begin{array}{l}\text { Any adverse events from performing the index tests or the reference } \\
\text { standard. }\end{array}$ & na \\
\hline Estimates & 21 & $\begin{array}{l}\text { Estimates of diagnostic accuracy and measures of statistical uncertainty } \\
\text { (e.g. } 95 \% \text { confidence intervals). }\end{array}$ & $\begin{array}{l}\text { 8-10 } \\
\text { Figure } 3\end{array}$ \\
\hline
\end{tabular}




\begin{tabular}{|l|c|l|l|l|}
\hline & 22 & $\begin{array}{l}\text { How indeterminate results, missing data and outliers of the index tests } \\
\text { were handled. }\end{array}$ & $\begin{array}{l}6, \\
\text { Figure } 1\end{array}$ \\
\hline & 23 & $\begin{array}{l}\text { Estimates of variability of diagnostic accuracy between subgroups of } \\
\text { participants, readers or centers, if done. }\end{array}$ & n.a. \\
\hline & 24 & Estimates of test reproducibility, if done. & na \\
\hline DISCUSSION & 25 & Discuss the clinical applicability of the study findings. & $11-14$ \\
\hline
\end{tabular}


Figure Legends:

Figure 1: Patient flow chart

Figure 2: Boxplots showing (a) baseline PENK, (b) day 1 PENK and (c) delta PENK levels in patients with or without contrast-induced AKI.

Figure 3: Receiver operating characteristic curves displaying the diagnostic accuracy of baseline PENK, day 1 PENK and delta PENK for the early detection of contrastinduced AKI 